\title{
Hydrodynamic Galaxy Cluster Simulations: a challenge for physics, parallel computing and visualisation
}

Wolfgang Kapferer*

Inst. for Astroph., Innsbruck, Austria

E-mail: wolfgang.e.kapferer@uibk.ac.at

W. Domainko, M. Mair, S. Schindler, E. van Kampen, S. Kimeswenger, M. Mair, Inst. for Astroph., Innsbruck, Austria

\section{Breitschwerdt}

Inst. for Astronomy, Vienna, Austria

\begin{abstract}
$\mathrm{N}$-body/hydrodynamic numerical simulations of galaxy clusters including the interaction of the intra-cluster medium (ICM) with the galaxies are one of the outstanding challenges in computational astrophysics. We include metal enrichment processes like galactic winds and ram-pressure stripping. Galactic winds are calculated with an additional code which takes into account cosmic rays as well as magnetic fields for supernovae driven winds. To treat higher star formation rates due to galaxy galaxy interactions smoothed particle hydrodynamic simulations are performed. This combination of different techniques allows us to determine the enrichment efficiency of different processes. Additionally, methods for the investigation of huge 3 dimensional data sets with state of the art visualisation techniques are shown.
\end{abstract}

Baryons in Dark Matter Halos

5-9 October 2004

Novigrad, Croatia

* Speaker. 


\section{Introduction}

From X-ray spectra it is evident that the ICM contains metals [4]. As heavy elements are only produced in stars the processed material must have been ejected by cluster galaxies into the ICM. Possible transport processes are ram pressure stripping [5], galactic winds [3], galaxy-galaxy interactions or jets from AGNs. In this work we concentrate on the effects of galactic winds of quiet, non-starburst, disk galaxies. Former publications on galactic winds [6] found that mass outflow occurs only at very high gas temperatures of several $10^{6} \mathrm{~K}$. For non-starburst galaxies, like our Milky Way, the temperature of the Hot Interstellar Medium (HIM) is below several $10^{6} \mathrm{~K}$, therefore radiative cooling prevents a continuous mass loss. This would lead to the conclusion that only active galaxies with an on-going starburst can enrich the ICM with metals due to thermally driven galactic winds. Models including ideal coupling of Cosmic Rays (CRs) and the HIM with self-excited Alvén waves (i.e. no diffusion), show that for a variety of parameters stationary outflow solutions do exist [1]. We calculate the mass loss rates due to galactic winds for quiet, i.e. nonstarburst, disk galaxies. The results from the models act as an input for our combined N-body hydrodynamic code which calculates the chemo-dynamical evolution of the ICM.

\section{The Simulations}

To simulate large-scale structure formation we use a $\Lambda$ CDM N-body tree code. Applying an additional semi-numerical model for galaxy formation [8] we derive the formation and evolution of the cluster galaxies. The gas is treated with a PPM (piecewise parabolic method) hydrodynamic grid code [2]. To obtain high resolution in the cluster center the hydrodynamic code uses a fixed mesh refinement [7]. The simulations are covering a volume of $(20 \mathrm{Mpc})^{3}$ by four nested grids so that the resolution in the center is three times higher than in the outer parts (Fig.1).

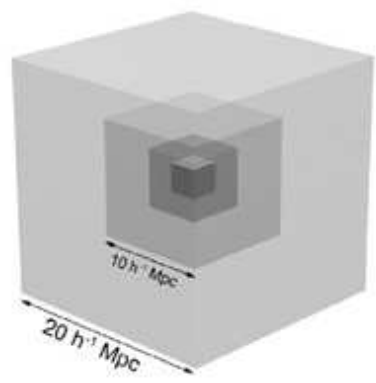

Figure 1: Nested grid topology. Each grid has the same number of cells, which leads to the highest resolution in the center of the simulation.

\section{The Galactic Wind Algorithm}

The mass-loss rate due to supernova driven galactic winds is calculated with a code developed by Breitschwerdt et al. (1991) [1]. The algorithm calculates for a given galaxy the mass-loss rate and wind properties like the velocity of the ejected matter as a function of distance to the galaxy or the pressure flow. As an input for the wind code galaxy parameters like halo mass, disc mass, 
spin parameter, scale length of the components, temperature distribution of the ISM, magnetic field strength and density distribution as well as stellar density distribution are required. All those properties are calculated within the semi-numerical model for galaxy evolution. In order to save computing time we performed parameter studies. The results are sumerised in a look-up table. One example of such a table is shown in Fig. 2. The mass loss rate is plotted as a function of the spin parameter and the mass of the disc. In this example the halo mass of $3 \times 10^{11} \mathrm{M}_{\odot}$ is assumed to be constant for all model galaxies.

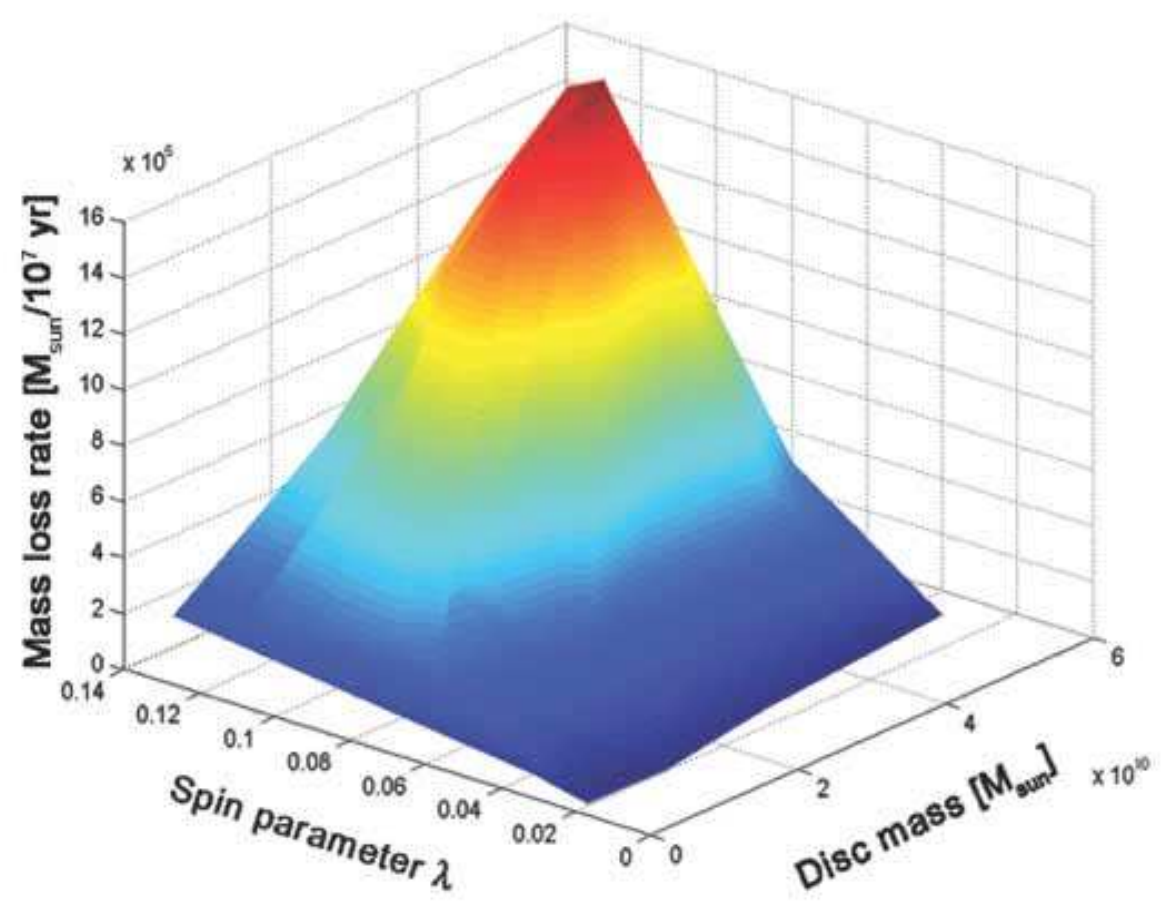

Figure 2: Mass loss rate as a function of disc mass and spin parameter for a given constant halo mass of $3 \times 10^{11} \mathrm{M}_{\odot}$.

The larger the disc mass and the spin parameter the higher is the mass loss rate. The extent of the disc increases with increasing spin parameter and therefore the probability for supernova explosions at large radii increases as well.

\section{First results}

In figure 3 the ejected matter due to galactic winds and the mixing with the ICM is shown. In the upper panel a temperature map (left) and a metallicity map (right) is given. The upper panel belongs to snapshot $\mathrm{z}=1$, whereas the lower panel shows the same quantities at $\mathrm{z}=0$. Each map has a side length of $10 \mathrm{Mpc}$. In the central region of the cluster the mixing of the ejected material with the ICM is more efficient than in the outer regions of the cluster. As the density of the ICM increases towards the cluster center the winds will be suppressed within a region of several $100 \mathrm{kpc}$ 
around the center due to the ICM pressure. This area can increase to sizes of $1 \mathrm{Mpc}$, depending on the mass of the model cluster. In the outer regions of the cluster where the density decreases galactic winds are more efficient to enrich the ICM than ram pressure stripping, see Domainko et al. (this volume).

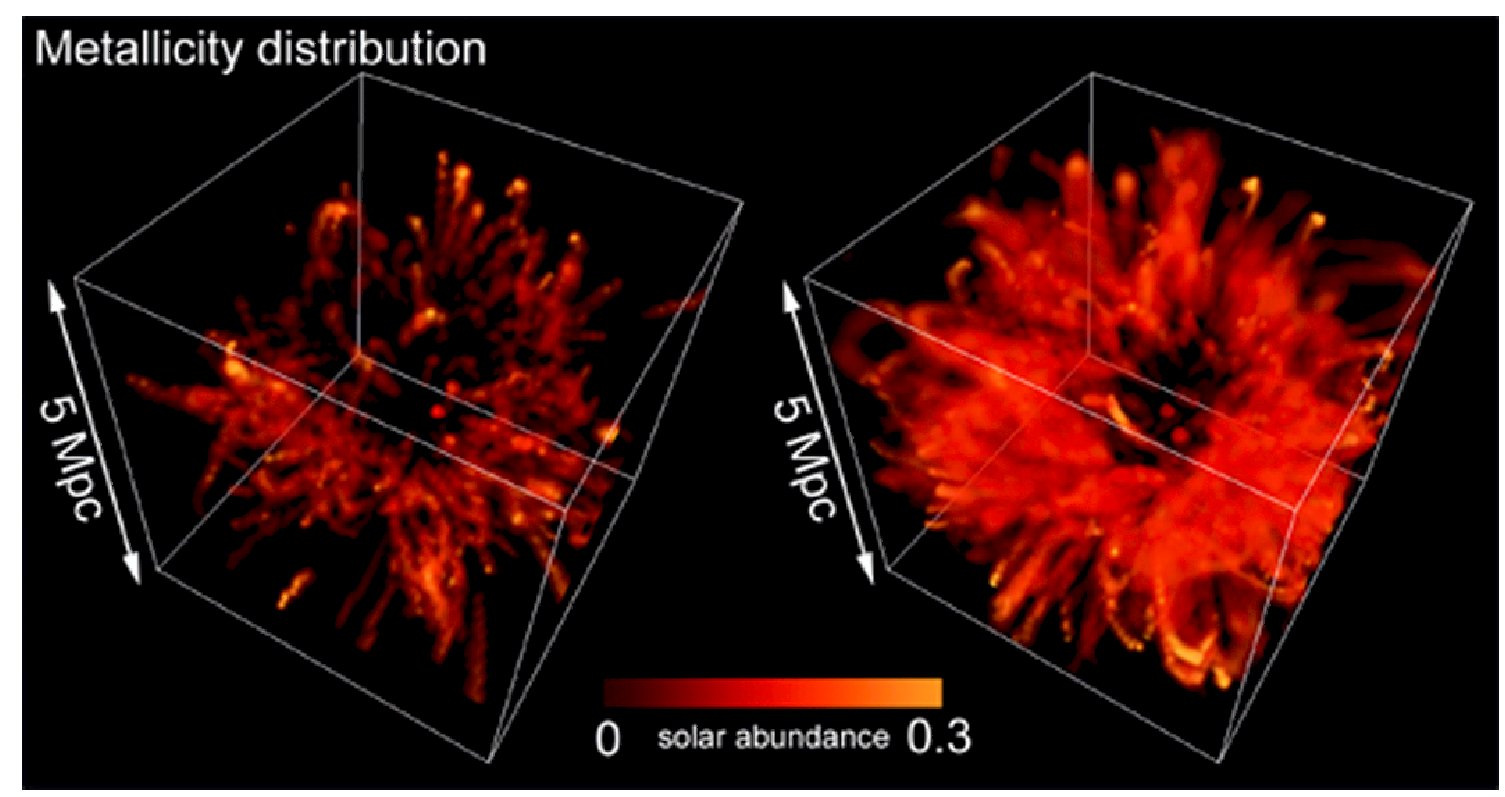

Figure 3: The left image displays the 3D metallicity distribution of the ICM for a model galaxy cluster at $\mathrm{z}=1$. The right images shows the same quantity in the same volume as the left image for $\mathrm{z}=0$. Each cube has $10 \mathrm{Mpc}$ on a side.

\section{References}

[1] Breitschwerdt, D., McKenzie, J.F., Völk, H.J., Galactic Winds, A\&A, 245, 79, 1991

[2] Colella, P., Woodward, P. R. The Piecewise Parabolic Method (PPM) for Gas Dynamical Simulations. J. Comput. Phys., 54, 174, 1984.

[3] De Young, D. S., On the origin and evolution of iron-enriched gas in clusters of galaxies. ApJ 223, 47, 1987.

[4] Fukazawa, Y. Makishima, K., Tamura, T., et al. ASCA Measurements of Silicon and Iron Abundances in the Intracluster Medium. PASJ 50, 187, 1998.

[5] Gunn, J. E., Gott J. R. III, On the Infall of Matter Into Clusters of Galaxies and Some Effects on Their Evolution. ApJ, 176, 1, 1972.

[6] McKee, C.F., Ostriker, J.P., A theory of the interstellar medium - Three components regulated by supernova explosions in an inhomogeneous substrate, ApJ 218, 148,1977

[7] Ruffert, M. Collisions between a white dwarf and a main-sequence star. II - Simulations using multiple-nested refined grids. A\&A, 265, 82, 1992.

[8] van Kampen, E., Jimenez, R., Peacock, J. A., Overmerging and mass-to-light ratios in phenomenological galaxy formation models. MNRAS, 310, 43, 1999. 